Journal for ImmunoTherapy of Cancer

\title{
Intracerebral administration of CTLA-4 and PD-1 immune checkpoint blocking monoclonal antibodies in patients with recurrent glioblastoma: a phase I clinical trial
}

Johnny Duerinck, ${ }^{1}$ Julia Katharina Schwarze (1) , ${ }^{2}$ Gil Awada (i) , ${ }^{2}$ Jens Tijtgat, ${ }^{2}$ Freya Vaeyens, ${ }^{3}$ Cleo Bertels, ${ }^{2}$ Wietse Geens (1) , ${ }^{1}$ Samuel Klein, ${ }^{1}$ Laura Seynaeve, ${ }^{4}$ Louise Cras, ${ }^{5}$ Nicky D'Haene, ${ }^{6}$ Alex Michotte, ${ }^{4,5}$ Ben Caljon, ${ }^{3}$ Isabelle Salmon, ${ }^{6}$ Michaël Bruneau, ${ }^{1}$ Mark Kockx, ${ }^{7}$ Sonia Van Dooren, ${ }^{3}$ Anne-Marie Vanbinst, ${ }^{8}$ Hendrik Everaert, ${ }^{9}$ Ramses Forsyth, ${ }^{5}$ Bart Neyns (i) ${ }^{2}$

To cite: Duerinck J,

Schwarze JK, Awada G, et al. Intracerebral administration of CTLA- 4 and PD- 1 immune checkpoint blocking monoclonal antibodies in patients with recurrent glioblastoma: a phase I clinical trial. Journal for ImmunoTherapy of Cancer 2021;9:e002296. doi:10.1136/ jitc-2020-002296

- Additional supplemental material is published online only. To view, please visit the journal online (http://dx.doi.org/10. 1136/jitc-2020-002296).

JD and JKS contributed equally.

JD and JKS are joint first authors.

Accepted 13 April 2021

Check for updates

(C) Author(s) (or their employer(s)) 2021. Re-use permitted under CC BY-NC. No commercial re-use. See rights and permissions. Published by BMJ.

For numbered affiliations see end of article.

Correspondence to

Bart Neyns;

bart.neyns@uzbrussel.be

\section{ABSTRACT}

Background Patients with recurrent glioblastoma (rGB) have a poor prognosis with a median overall survival (OS) of 30-39 weeks in prospective clinical trials. Intravenous administration of programmed cell death protein 1 and cytotoxic T-lymphocyte-associated antigen 4 inhibitors has low activity in patients with rGB. In this phase I clinical trial, intracerebral (IC) administration of ipilimumab (IPI) and nivolumab (NIVO) in combination with intravenous administration of NIVO was investigated.

Methods Within 24 hours following the intravenous administration of a fixed dose $(10 \mathrm{mg})$ of NIVO, patients underwent a maximal safe resection, followed by injection of IPI (10 mg; cohort-1), or IPI (5 mg) plus NIV0 (10 mg; cohort-2) in the brain tissue lining the resection cavity. Intravenous administration of NIVO (10 mg) was repeated every 2 weeks (max. five administrations). Next generation sequencing and RNA gene expression profiling was performed on resected tumor tissue.

Results Twenty-seven patients were enrolled (cohort-1: $n=3$; cohort-2: $n=24$ ). All patients underwent maximal safe resection and planned IC administrations and preoperative NIV0. Thirteen patients (cohort-1: $n=3$; cohort-2: $n=10$ ) received all five postoperative intravenous doses of NIVO. In cohort-2, 14 patients received a median of 3 (range 1-4) intravenous doses. Subacute postoperative neurological deterioration $(n=2)$ was reversible on steroid treatment; no other central nervous system toxicity was observed. Immune-related adverse events were infrequent and mild. GB recurrence was diagnosed in 26 patients (median progression-free survival (PFS) is 11.7 weeks (range 2-152)); 21 patients have died due to progression. Median OS is 38 weeks (95\% Cl: 27 to 49) with a 6-month, 1-year, and 2-year OS-rate of, respectively, 74.1\% (95\% Cl: 57 to 90), $40.7 \%$ (95\% Cl: 22 to 59), and $27 \%$ (95\% Cl: 9 to 44). OS compares favorable against a historical cohort (descriptive Log-Rank $p>0.003$ ). No significant difference was found with respect to PFS (descriptive Log-Rank test $p>0.05$ ). A higher tumor mRNA expression level of
B7-H3 was associated with a significantly worse survival (multivariate Cox logistic regression, $\mathrm{p}>0.029$ ). Conclusion IC administration of NIVO and IPI following maximal safe resection of rGB was feasible, safe, and associated with encouraging $0 \mathrm{~S}$.

Trial registration NCT03233152.

\section{INTRODUCTION}

Glioblastoma is the most common malignant primary brain tumor in adults. The current standard of care consists of maximal safe neurosurgical resection or diagnostic biopsy, followed by postoperative radiation therapy plus concomitant and adjuvant temozolomide chemotherapy. Despite this upfront multimodality treatment, progression of disease within less than 9 months is seen in more than half of glioblastoma patients and 5 year survival is less than $10 \%{ }^{1}$

Salvage treatment for patients with recurrent glioblastoma has typically been disappointing with a median progression-free survival (PFS) and a median overall survival (OS) ranging from 10 to 17 weeks and from 30 to 39 weeks, respectively; no randomized clinical trial to date has been able to demonstrate improvement of OS, underlining the urgent need for new active treatment options. ${ }^{2-5}$

The role of surgery for glioblastoma patients presenting with a resectable recurrence remains controversial. Several observational trials have shown that increased extent of resection in the recurrent setting leads to prolonged survival. ${ }^{6-8}$ However, a pooled analysis of patients with recurrent glioblastoma who participated in phase II clinical trials sponsored by the North American Brain 
Tumor Consortium ( $\mathrm{n}=758)$ showed no difference in survival between patients who underwent surgery at recurrence and those who received pharmacological therapy. ${ }^{9}$

Immune checkpoint inhibition by means of therapeutic monoclonal antibodies $(\mathrm{mAb})$ targeting the programmed cell death protein 1 (PD-1, CD279), programmed deathligand 1 (PD-L1, CD274) or cytotoxic T-lymphocyteassociated antigen 4 (CTLA-4, CD152) is effective in many types of solid and hematological malignancies. ${ }^{10}$ The potential of immune checkpoint inhibition as a treatment strategy for glioblastoma was also demonstrated in several preclinical glioblastoma models. ${ }^{1-17}$

A phase I trial evaluating the intravenous administration of the anti-PD-1 checkpoint inhibitor nivolumab as a single agent or in combination with different dose levels of the CTLA-4-blocking mAb ipilimumab in patients with recurrent glioblastoma indicated that nivolumab as a single agent was well tolerated but the combination of nivolumab and ipilimumab was associated with up to $30 \%$ treatment-related adverse events (TRAE) leading to treatment discontinuation. The tolerability of the combination was determined by the dose of ipilimumab. Interestingly, in two patients who were initially suspected to have progressed based on neuroradiological assessments and subsequently underwent a neurosurgical resection, histopathological examination revealed large aggregates of immune cells, but no viable tumor. ${ }^{18}$

However, the phase III CheckMate-143 trial showed that nivolumab treatment in patients with recurrent glioblastoma was largely ineffective when compared with patients treated with the anti-vascular endothelial growth factor A (VEGF-A) mAb bevacizumab. Objective response rate (ORR) was lower $(7.8 \%$ vs $23.1 \%)$, PFS was also inferior (median PFS of 1.5 months vs 3.5 months) while OS was identical. It was noted that responses obtained with nivolumab were more durable compared with bevacizumab (11.1 months vs 5.3 months) ${ }^{19}$ Similarly, the PD-1blocking mAb pembrolizumab was not shown effective in patients with recurrent glioblastoma. ${ }^{20}$ In a phase II study comparing pembrolizumab monotherapy with the combination of pembrolizumab plus bevacizumab for patients with recurrent glioblastoma, no significant antitumoral activity was observed in the monotherapy arm and the PFS obtained with the combination therapy was comparable to that of historical data obtained with bevacizumab monotherapy. ${ }^{21}$

More recently, the phase III trial (CheckMate-498), comparing nivolumab plus radiation therapy with the standard combination of radiation therapy plus temozolomide chemotherapy in patients with newly diagnosed glioblastoma without methylated O6-methylguanine-DNA methyltransferase (MGMT), failed to meet its endpoint of improved OS. ${ }^{22}$ Likewise, the CheckMate- 548 phase III trial (NCT02667587) evaluating the addition of nivolumab to standard radiation therapy plus temozolomide chemotherapy in patients with newly diagnosed glioblastoma with MGMT-methylation failed to meet its endpoint of improved PFS.
The low activity of PD-1 blocking mAb as a monotherapy for recurrent glioblastoma could be expected taking into account the absence of predictive biomarkers that correlate with the activity of antiPD-1 therapy in other tumor types (such as a high tumor mutation burden (TMB) and/or interferongamma (IFN- $\gamma$ ) signature) ${ }^{23}$ Glioblastomas are typically characterized by a low TMB and a lack of strong baseline immune infiltration of the tumor microenvironment (TME). In addition, the central nervous system is often referred to as a site of 'immune privilege' due to the presence of the blood-brain barrier that shields off the entrance of mAb from the systemic circulation. ${ }^{24}$ Nevertheless, children with hypermutant glioblastoma resulting from germline biallelic mismatch repair deficiency were found to benefit from anti-PD-1 monotherapy. ${ }^{25}$ Furthermore, in melanoma patients with brain metastases, the combination of anti-PD-1 and anti-CTLA- 4 mAb resulted in intracranial efficacy suggesting that these $\mathrm{mAb}$ were able to block their receptor within the TME of these intracranial metastases. ${ }^{26}$ In adult glioblastoma patients, the buildup of an antitumor T-cell repertoire has been demonstrated, but these antitumor $\mathrm{T}$ cells were exhausted and tend to sequester in the bone marrow. ${ }^{27} 28$

The potential for (re-)invigorating the antitumor immune response in patients with recurrent glioblastoma was recently demonstrated in studies using neoadjuvant PD-1 blockade. Neoadjuvant administration of pembrolizumab preceding surgical intervention was associated with the upregulation of T-cell and IFN- $\gamma$-related gene expression in immune cells and the downregulation of cell-cycle-related gene expression within the tumor. Notwithstanding the small patient numbers in this trial, there was also a significantly improved survival in patients treated in the neoadjuvant arm compared with those receiving adjuvant treatment (13.2 months vs 6.3 months)..$^{29-31}$

Across solid tumor types, the combination of antiCTLA-4 and PD-1 mAb has resulted in improved tumor response rates and PFS compared with single agent anti-PD-1, but at the cost of a significantly higher rate of immune-related adverse events (irAE). In animal models, intratumoral administration of CTLA-4 blocking mAb offers a better risk-to-benefit ratio compared with systemic administration. ${ }^{32} 33$ Intratumoral administration of ipilimumab in extracranial metastases of solid tumors was recently found to be tolerable and associated with early indications of activity. ${ }^{345}$

In order to explore the potential of the combination of CTLA-4 plus PD-1 blockade in patients with recurrent glioblastoma, we conducted a phase I clinical trial investigating the intracerebral administration of ipilimumab with or without nivolumab in combination with intravenous low dose nivolumab in patients with a resectable glioblastoma recurrence. 


\section{METHODS}

\section{Study design and patient eligibility}

This single-center, open-label phase I clinical trial was conducted at the Universitair Ziekenhuis Brussel (UZ Brussel, Brussels, Belgium).

Patients were eligible if they were aged $\geq 18$ years and had a recurrence of a previously histologically confirmed glioblastoma (or a lower grade glioma that had transformed to a WHO grade 4 glioma), and that was amenable for a gross-total resection (with an acceptable risk for postoperative neurological deficits).

Progression of disease needed to be documented following prior treatment that at least included radiation therapy and temozolomide chemotherapy. At baseline, a measurable tumor recurrence on gadoliniumenhanced T1 magnetic resonance imaging (MRI) and on 18-fluoroethyl-L-tyrosine positron-emission tomography/ computed tomography $\left({ }^{18}\right.$ F-FET-PET/CT $)$ was required.

Patients requiring oral corticosteroids exceeding a maximal daily equivalent dose of methylprednisolone (MPS) $\geq 8 \mathrm{mg}$ or dexamethasone $\geq 1.5 \mathrm{mg}$ per day for at least 7 days prior to enrolment were considered ineligible. Eastern Cooperative Oncology Group (ECOG) Performance Status had to be $\leq 2$ and adequate hepatic, renal and bone marrow function was required. Patients were excluded if they had received prior immunotherapy with an anti-PD-1, -PD-L1, or -CTLA-4 mAb or any other drug specifically targeting immune checkpoints or if they were treated with immunosuppressive drugs (eg, in case of organ transplant) with the exception of topical steroids or oral steroids at the maximum daily dose mentioned previously in this paragraph. Pre-existing active autoimmune disease, prior immunodeficiency syndromes, persisting toxicities from prior therapies, diagnosis of any other malignancy within 5 years prior to enrolment, bleeding/thrombotic disorders or problematic wound healing also excluded the patient from participation. All participants provided written informed consent.

\section{Treatment plan}

Twenty-fourhours prior to surgical intervention, $10 \mathrm{mg}$ of nivolumab (Opdivo ${ }^{\circledR}$, Bristol Myers Squibb (BMS)) was administered by intravenous infusion over $15 \mathrm{~min}$. Surgical resection was performed under 5-Amino-Levulinic-Acid (5-ALA) fluorescence. At the end of the surgical intervention, after careful hemostasis was achieved, the brain tissue lining the resection cavity was injected manually (by multiple $100 \mu \mathrm{L}$ administrations) with $2 \mathrm{~mL}$ of ipilimumab (Yervoy ${ }^{\circledR}$, BMS, $10 \mathrm{mg}$ ) in cohort- 1 , and $1 \mathrm{~mL}$ of nivolumab $(10 \mathrm{mg})$ plus $1 \mathrm{~mL}$ of ipilimumab (5 mg) in cohort-2. The 20 injections were evenly distributed to cover the entire resection cavity wall but avoiding any nearby functional areas; tractography of the white matter tracts loaded into the navigation system was used to evaluate this. A tuberculin needle inserted to a depth of 3-5 mm was used, and the goal on injection was to obtain a slight swelling of the tissue and no evacuation of the compound into the resection cavity.
Thereafter, intravenous administrations of nivolumab (at a fixed dose of $10 \mathrm{mg}$ ) were repeated every 2 weeks for a total maximum of five administrations. Treatment was discontinued in case of confirmed progression of disease, unacceptable toxicity or patient refusal to continue study treatment. Patients could continue study treatment following the first documentation of disease progression if the investigator considered this to be in the best interest of the patient.

\section{Assessment}

Throughout the study, patients were evaluated every 2 weeks with a clinical examination and blood analysis. Gadolinium-enhanced MRI was performed on the first day postoperatively and every 6 weeks thereafter. Follow-up imaging with ${ }^{18}$ F-FET-PET/CT was scheduled as clinically indicated to complement MRI results. Tumor responses and progression of disease were defined according to the immunotherapy response assessment for neuro-oncology criteria.

\section{Analysis of resected tumor tissues}

Tumor tissue was collected from all patients at the time of their surgical intervention. H\&E staining and immunohistochemistry (IHC) analyses were performed using formalin-fixed, paraffin-embedded (FFPE) tumor tissue. CD8 SP57 (Roche, Basel, Switzerland) and PD-L1 22C3 (Agilent, CA, USA) antibodies were used for biomarker analysis. Tumor PD-L1 expression was scored by a pathologist for the frequency of tumor cells with membrane staining of PD-L1 (ie, patients with PD-L1 expression levels $\geq 1 \%$ were defined as those with $\geq 1$ PD-L1-positive tumor cells within a field of 100 evaluable cells). For the purpose of IHC staining for B7-H3, FFPE sections were deparaffinized and stained with a validated chromogenic IHC assay based on an anti-B7-H3 primary Rabbit monoclonal antibody (SP-265, Spring Biosciences). The detection was done by a Dako EnVision+System HRP Labeled Polymer Anti-Rabbit on a DAKO Autostainer Link 48. MGMT promoter methylation analysis was performed by MethyLight as previously described. ${ }^{36}{ }^{37}$ Next-generation sequencing (NGS) to reveal somatic mutations was performed on FFPE-extracted-DNA using an accredited in-house capture-based comprehensive gene panel (ACVR1, ATRX, BRAF, CDKN2A, CTNNB1, EGFR, ERBB3, H3F3A, H3F3B, HIST1H3B, HIST1H3C, IDH1, IDH2, MET, NF1, NTRK1, NTRK2, NTRK3, PDGFRA, PIK3CA, PTEN, SMO, TERT, TP53) on Illumina NovaSeq6000. A validated homebrew bioinformatics pipeline provided variants that were biologically and clinically classified according to the Belgian ComPerMed (v1) guidelines. ${ }^{38}$

Total RNA was isolated from FFPE-pretreated tumor biopsies. Initially, tumor samples from 21 patients were selected; total RNA extraction was sufficient for 18 tumor samples. Gene expression profiling (GEP) of 770 genes was performed using the NanoString PanCancer IO 360 Panel (NanoString Technologies) according to the 
manufacturer's instructions. Released tags were quantified in a standard nCounter analysis system. Biological signatures and scores based on the GEP were provided by NanoString Technologies.

\section{Objectives and statistical analysis}

The primary objectives of the trial were to establish safety and feasibility of the experimental treatment regimen. Primary endpoints were TRAE according to the National Cancer Institute (NCI) Common Terminology Criteria for Adverse Events (CTCAE) version 5 and treatment disposition for nivolumab and ipilimumab.

Secondary endpoint was OS analyzed by Kaplan-Meier estimator.

Initially, the sample size for cohort- 1 was determined according to a classical phase I 3+3 design. Following the first three patients treated in cohort 2 , the trial was amended to initially expand this cohort to 12 patients and by a second amendment to recruit a final of 24 patients. Statistical analyses were performed using SPSS Statistics V.26.0 (IBM, Chicago, USA).

\section{RESULTS}

\section{Patient baseline characteristics}

Between November 16, 2016 and August 8, 2019, 27 patients were enrolled and initiated study treatment, 3 and 24 patients in cohort-1 and cohort-2, respectively. The median age was 55 years (range 38-74). Most patients $(81 \%)$ were initially diagnosed with a WHO grade 4 glioma (glioblastoma) and (85\%) had been treated at first diagnosis by a surgical intervention followed by adjuvant radiation therapy $(30 \times 2 \mathrm{~Gy})$ with concomitant and adjuvant temozolomide chemotherapy. Six patients had progressed on a prior systemic chemotherapy administered at first recurrence. At baseline, most patients (89\%) had an ECOG PS of $0-1$ without need for high doses of corticosteroids ( $\leq 8 \mathrm{mg}$ of MPS per day); a waver was given to four patients who were treated with more than $8 \mathrm{mg}$ MPS daily $(16 \mathrm{mg} /$ day in one patient, $2 \times 16 \mathrm{mg} /$ day in two patients, and $2 \times 32 \mathrm{mg} /$ day in one patient) at the time of enrolment because it was anticipated that resection of the recurrent glioblastoma would alleviate the need for corticosteroid treatment. The majority of gliomas were known to be IDH1 wild type $(\mathrm{n}=25 ; 93 \%)$. No loss of heterozygosity $(\mathrm{LOH})$ for $1 \mathrm{p} / 19 \mathrm{q}$ was found in the eight gliomas analyzed ( $\mathrm{LOH}$ for $1 \mathrm{p} / 19 \mathrm{q}$ was not analyzed in 19 patients $(70 \%))$. All patients presented with a progressive gadolinium-enhancing tumor mass that was also visualized by ${ }^{18}$ F-FET-PET/CT of the brain. Baseline patient characteristics and prior therapies are summarized in table 1 .

\section{Patient disposition and drug exposure}

All patients received a $10 \mathrm{mg}$ dose of in travenous nivolumab 24 hours before the surgical intervention during which they underwent resection of the gadolinium-enhanced tumor mass guided by 5-ALA fluorescence. Gross total
Table 1 Baseline patient demographics and prior therapy

\begin{tabular}{|c|c|c|}
\hline & & $\begin{array}{l}N(\%) \\
(n=27)\end{array}$ \\
\hline Age & Median (years; range) & $55(38-74)$ \\
\hline \multirow[t]{2}{*}{ Sex } & Male & $17(63)$ \\
\hline & Female & $10(37)$ \\
\hline \multirow[t]{3}{*}{ ECOG PS } & 0 & $10(37)$ \\
\hline & 1 & $14(52)$ \\
\hline & 2 & $3(11)$ \\
\hline \multirow{3}{*}{$\begin{array}{l}\text { Primary } \\
\text { histopathologic } \\
\text { diagnosis }\end{array}$} & WHO Grade IV & $22(81)$ \\
\hline & WHO Grade III & $4(15)$ \\
\hline & WHO Grade II & $1(4)$ \\
\hline \multirow{2}{*}{$\begin{array}{l}\text { IDH1/2 mutation } \\
\text { status }\end{array}$} & Mutant & $2(7)$ \\
\hline & Wild-type & $25(93)$ \\
\hline \multirow{3}{*}{$\begin{array}{l}\text { MGMT-promotor } \\
\text { methylation status }\end{array}$} & Methylated & $7(26)$ \\
\hline & Unmethylated & $13(48)$ \\
\hline & Unknown & $7(26)$ \\
\hline \multirow[t]{3}{*}{$1 p / 19 q$ codeletion } & Yes & $0(0)$ \\
\hline & No & $8(30)$ \\
\hline & Unknown & $19(70)$ \\
\hline \multirow{2}{*}{$\begin{array}{l}\text { Prior surgical } \\
\text { intervention }\end{array}$} & Resection & $27(100)$ \\
\hline & Biopsy & $0(0)$ \\
\hline \multirow{3}{*}{$\begin{array}{l}\text { Prior therapy for } \\
\text { primary diagnosis } \\
\text { of glioma }\end{array}$} & $\begin{array}{l}\text { Surgery+concomitant RT/ } \\
\text { TMZ+adjuvant TMZ }\end{array}$ & $23(85)$ \\
\hline & Surgery only & $2(7)$ \\
\hline & Surgery+RT & $2(7)$ \\
\hline \multirow{7}{*}{$\begin{array}{l}\text { Therapy for prior } \\
\text { recurrent disease }\end{array}$} & Surgery only & $2(7)$ \\
\hline & Surgery+RT+TMZ & $2(7)$ \\
\hline & Surgery+RT & $1(4)$ \\
\hline & Surgery+TMZ & $1(4)$ \\
\hline & Surgery+TMZ+lomustine & $1(4)$ \\
\hline & Surgery+irinotecan & $1(4)$ \\
\hline & $\mathrm{RT}+\mathrm{TMZ}$ & $1(4)$ \\
\hline \multicolumn{2}{|c|}{ Median of prior systemic therapies } & $3(1-4)$ \\
\hline
\end{tabular}

$1 p / 19 q$ codeletion: deletion of the short arm of chromosome 1 and long arm of chromosome 19.

ECOG, Eastern cooperative oncology group; IDH1/2, isocitrate dehydrogenase 1/2; MGMT, methylated O6-methylguanine-DNA methyltransferase; $\mathrm{RT}$, radiotherapy; $\mathrm{TMZ}$, temozolomide.

resection was achieved in 10 of 27 patients (37\%), 17 patients $(63 \%)$ underwent a subtotal resection (figure 1). All patients received the predefined dose of intracerebral ipilimumab (cohort-1), or ipilimumab plus nivolumab (cohort-2), administered within the brain tissue lining the resection cavity at the end of the surgical intervention.

Thirteen patients (including all three patients treated in cohort-1; and 10 patients in cohort-2 $(41 \%))$ received all five planned postoperative intravenous doses of nivolumab. In 14 patients (52\%) from cohort-2, study treatment was stopped at an earlier point because of 


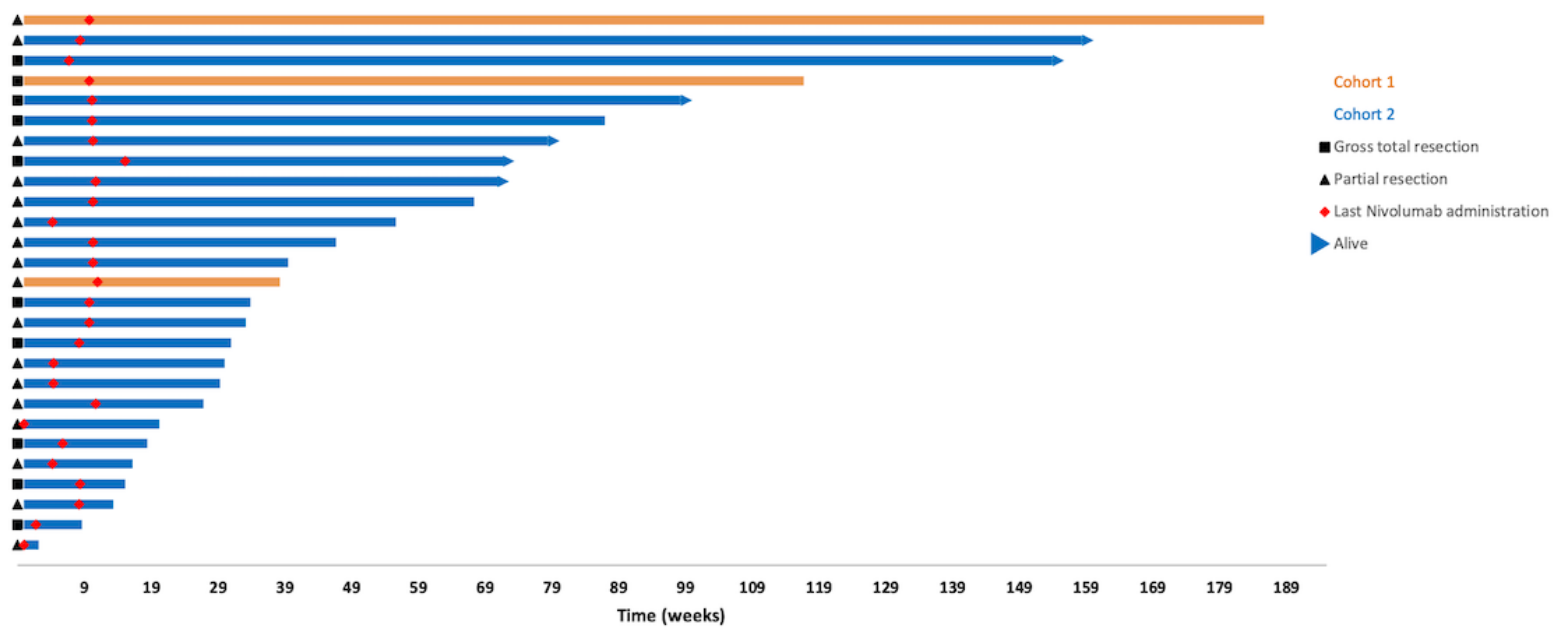

Figure 1 Swimmer plot representing the survival, surgical resection extent, and last nivolumab dosing per individual patient. Each bar represents an individual patient. Patients from cohort 1 and cohort 2 are depicted by, respectively, an orange or blue bar. Gross total resection and partial resection are depicted by black square and black triangle, respectively. Last administration of nivolumab is indicated by a red square. A blue arrowhead at the end of a bar indicates patients who are still alive.

symptomatic tumor progression; in these patients, a median number of 3 (range 1-4) intravenous doses were administered (figure 1 and online supplemental table 1).

\section{Safety}

The peroperative injections of ipilimumab (cohort1 ), or ipilimumab plus nivolumab (cohort-2) could be performed as planned without occurrence of any peroperative complications.

Postoperative fever $(\mathrm{n}=14$; grade $1 \quad(\mathrm{n}=12$; including all three patients from cohort-1), and grade $2(\mathrm{n}=2)$ ) was the only adverse event (AE) that was suspected of being related to the administration of ipilimumab and nivolumab. There was a low incidence of clinically significant grade $1 / 2 \mathrm{AE}$ that were suspected to be immunerelated: pruritus and rash $(\mathrm{n}=7$, and $\mathrm{n}=4$ respectively), hypothyroidism $(\mathrm{n}=2)$, a sarcoid-like reaction $(\mathrm{n}=1)$, and fatigue $(n=17)$. These AE did not require systemic corticosteroid treatment or other immunosuppressive therapy. The only grade $5 \mathrm{AE}$ was an infectious pneumonia that was considered not to be related to study treatment. Allcause AE occurring in more than two patients as well as $\mathrm{AE}$ of special interest are listed in online supplemental table 2. Lymphopenia was the most frequent abnormal blood value during study treatment and was observed in 25 of 27 patients ( $\geq$ grade $3, n=10$ ) (online supplemental table 3). In all patients, lymphopenia was present at baseline and was not considered to be related to the study treatment.

\section{Efficacy}

Confirmed tumor progression was documented in all except one male patient from cohort-2 (who remained free from progression 158 weeks after initiating study treatment). The initial postoperative evolution of this patient was characterized by a pseudoprogression on MRI of the brain (figure 2).

At the time of radiological progression, a total of three study patients underwent a new neurosurgical resection of the suspected lesion. In a first female patient, a growing cystic lesion located at the resection site was surgically explored. Anatomopathological analysis of the removed inflammatory membrane lining the cavity as well as biopsies taken of the surrounding brain tissue revealed no glioblastoma tumor cells, instead infiltration by inflammatory cells mainly composed of lymphocytes was found (figure 3). In an additional female patient from cohort-2 who underwent a complete resection of a suspected tumor recurrence 60 weeks after the initiation of study treatment, the resected tissue revealed no evidence of recurrent glioblastoma, but post-treatment changes characterized by inflammatory changes enriched with lymphocytes and histiocytes as well as siderophages with multinuclear giant cells (figure 3). This patient subsequently resumed immunotherapy and remains free from progression 98 weeks after initiating study treatment. In a third, male patient, the resected tissue was found to be composed of glioblastoma with focal infiltration by lymphocytes and histiocytes.

At the time of data-base lock, 21 patients (78\%) have died (all caused by glioblastoma progression). The median follow-up of the six (22\%) surviving patients is 108 weeks (range 67-158). The median PFS for the total study population was 11.7 weeks (95\% CI: 10 to 12; range 2-152) (figure 4A). Median OS is 38 weeks (95\% CI: 27 to 49 ) with a 6-month, 1-year, and 2-year OS-rate of, respectively, $74.1 \%$ (95\% CI: 57 to 90 ), $40.7 \%$ (95\% CI: 22 to 59 ), and $27 \%$ (95\% CI: 9 to 44) (figure 4B). For the purpose of comparing survival with a historical control 


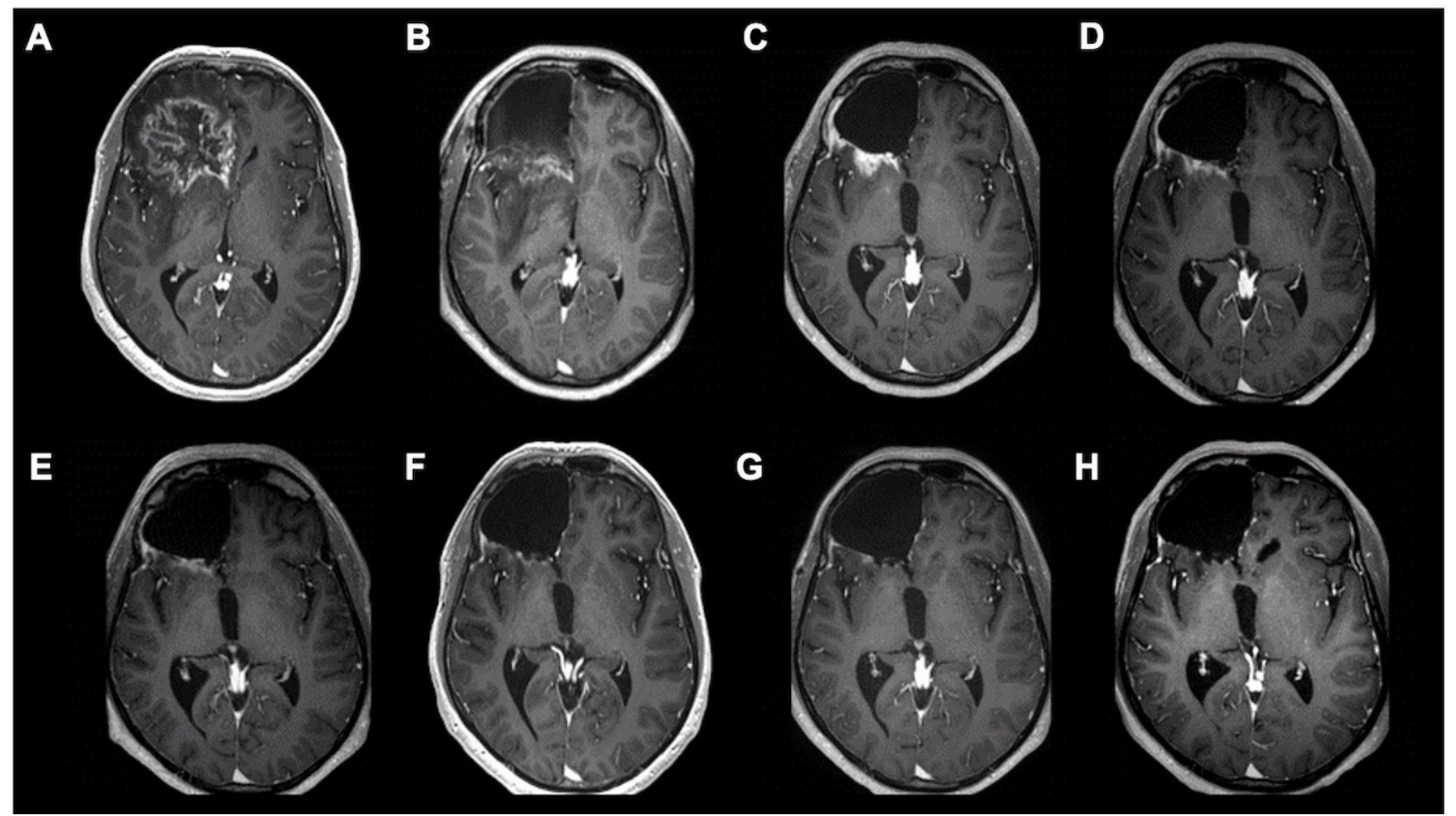

Figure 2 Case of a 42-year-old male patient with progressive disease of glioblastoma (IDH-1 wild type). The patient was diagnosed 4.5 years earlier with an anaplastic astrocytoma (WHO grade 3, IDH-1 wild type) of the left frontal lobe that was completely resected and treated with adjuvant radiation therapy with concomitant temozolomide and six cycles of adjuvant temozolomide. At first recurrence, 3 years later, was treated again with radiation therapy (60 Gy) with concomitant temozolomide and 6 cycles of adjuvant temozolomide. (A) Axial view of gadolinium-enhanced T1 MRI of the brain, 17 months after the first progression, the patient was enrolled in the study, underwent a frontal lobectomy with injection of ipilimumab (5 mg) and nivolumab $(10 \mathrm{mg})$ in the brain tissue lining the resection cavity. (B) Postoperative imaging reveals limited gadolinium enhancement of the brain tissue lining the resection cavity. $(\mathrm{C}-\mathrm{H})$ Axial images of gadolinium-enhanced T1 MRI of the brain were made with a 6 weeks interval. Postoperative gadolinium enhancement at the margins of the resection cavity decreases through time. The patient remains disease-free 2.7 years after initiating study treatment. IDH-1, isocitrate dehydrogenase-1.

group, a cohort of 469 Belgian patients with recurrent glioblastoma who were treated in three prospective phase II clinical trials (investigating axitinib, avelumab, and lomustine as investigational drugs), and a medical need program for bevacizumab was used. ${ }^{39-42}$ No significant difference was found with respect to PFS (descriptive Log-Rank test $\mathrm{p}>0.05$ ). OS compared favorably with the OS of the historical control group $(n=469)$, with a marked tendency for superior OS in patients with the longest survival and improved 1-year, 2-year, and 3-year survival estimates (descriptive Log-Rank test $\mathrm{p}>0.003$ ). Six-months, 1-year, and 2-year OS-rates in this historical control group were, respectively, $50.2 \%$ (95\% CI: 46 to 55 ), $18.3 \%$ (95\% CI: 15 to 22 ), and $5.3 \%$ (95\% CI: 3 to 8 ). When compared with the OS of a subpopulation of the historical control cohort composed of patients that were not in need of corticosteroid treatment $(n=27$; cohort- 1 of the GliAvAx study), our study population also had a significantly better OS (descriptive Log-Rank p>0.048). ${ }^{42}$

\section{Molecular tumor characteristics and correlation with survival}

All resected glioma tumor tissues were graded WHO grade 4 , at the exception of one patient in whom, despite the MRI features of a high-grade gadolinium-enhancing glioma recurrence, the tumor biopsy revealed a grade 2 glioma (this patient nevertheless had a relatively short PFS of 22 weeks).

NGS analysis of DNA extracted from the resected tumor tissue detected pathogenic mutations in 20 patients $(74 \%) ; 12(44 \%)$ had a TERT c. $-124 \mathrm{C}>\mathrm{T}$ mutation, 9 $(33 \%)$ a TP53 mutation, $6(22 \%)$ a NF1 mutation, and 2 $(7 \%)$ an IDH1 R132H mutation (table 2).

None of the recurrent glioblastomas with a IDH1 mutation was characterized by a $1 \mathrm{p} 19 \mathrm{q}$ co-deletion. Seven out of the 20 glioblastomas $(35 \%)$ that could be analyzed were characterized by MGMT-promotor hypermethylation. No significant correlation was found between the somatic mutation genotype, or MGMT-promotor methylation status and survival.

The immunophenotype was assessed by PD-L1 $(n=27)$, and CD8 score $(n=27)$, and NanoString IO 360 GEP scores $(n=18$; figure $5 \mathrm{~A})$. Immunohistochemical analysis has shown PD-L1 expression in seven patients. We did not observe a correlation between neither PD-L1 IHC and PD-L1 GEP score nor CD8 IHC score and CD8 GEP score.

Significant correlations between OS and the GEP score for NOS2, B7-H3, proliferation, CD45, JAK/STAT-loss, and immunoproteasome-score were found in univariate analysis. In a multivariate analysis, a higher tumor 

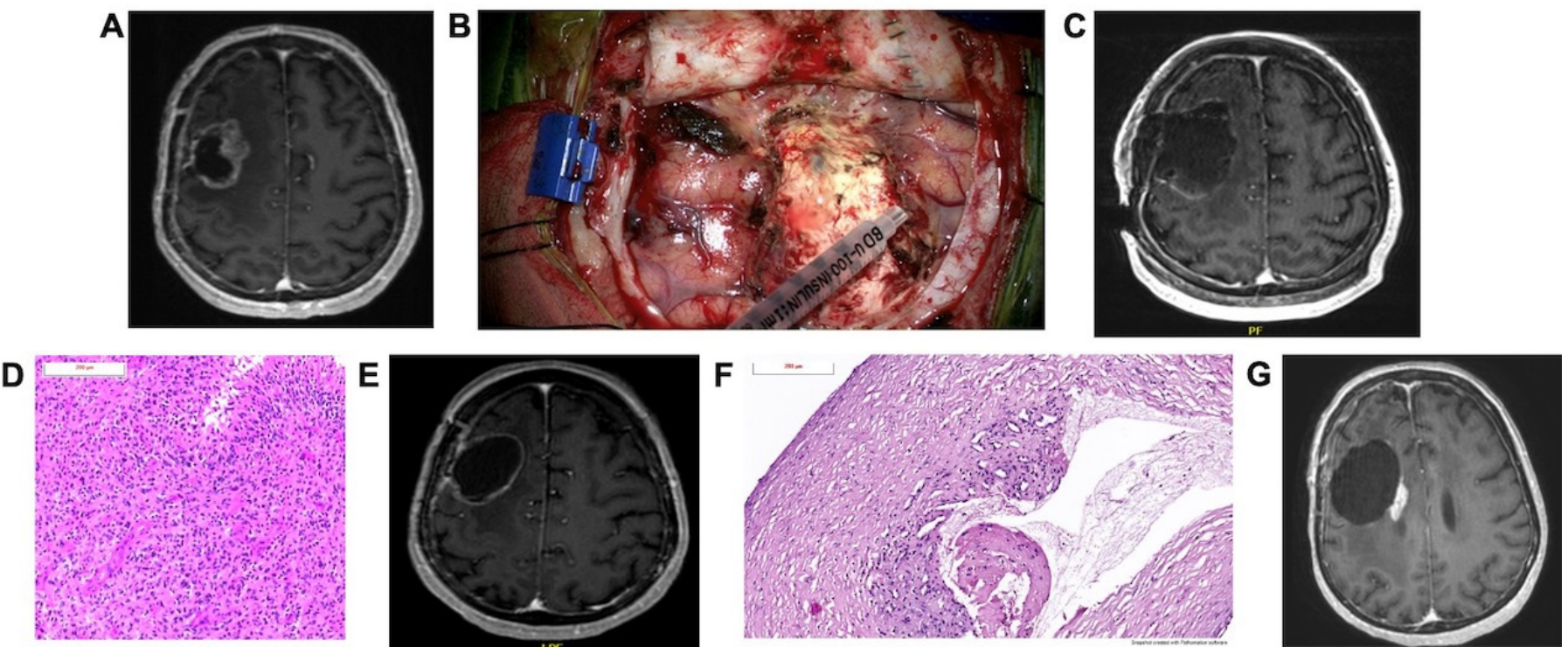

Figure 3 Case illustration of a 73-year-old female patient with progressive disease of glioblastoma (IDH-1 wild type) (A) Axial view of gadolinium-enhanced T1 MRI of the brain 8 months after a first complete resection followed by adjuvant radiation therapy $(30 \times 2$ Gy) with concomitant temozolomide chemotherapy and six cycles of adjuvant temozolomide showing progressive disease. (B) Photograph showing a perioperative image of injection of ipilimumab $(10 \mathrm{mg})$ in the brain tissue lining the resection cavity after complete resection of the tumor recurrence. (C) Postoperative axial view of gadolinium-enhanced T1 MRI of the brain showing no contrast captation. (D) Histopathological examination of the resected tissue with H\&E staining confirmed a WHO grade 4 glioma. (E) Axial view of gadolinium-enhanced T1 MRI of the brain 4 months after the initiation of study treatment (patient received all 4 intravenous nivolumab administrations), revealed a thickening of the gadolinium-enhanced lining of the resection cavity with increase in perilesional edema. (F) During a neurosurgical exploration, tissue lining the resection cavity was removed and a biopsy of the underlying brain was obtained. Histopathological examination (H\&E) revealed a collagenrich tissue with areas of necrosis, hemosiderin deposits, infiltrates of lymphocytic cells, thick-walled vessels with signs of fibrinoid necrosis. No evidence of glioblastoma cells was found. (G) Axial view of gadolinium-enhanced T1 MRI of the brain showing tumor progression 5 months later. The patient died of progressive disease 35 months after initiating study treatment. IDH-1, isocitrate dehydrogenase-1.

mRNA expression level of B7-H3 was the only GEP score associated with a significantly worse survival (multivariate Cox logistic regression, $\mathrm{p}>0.029$ ) (figure 4C). B7-H3 gene expression correlated well with immunohistochemical staining intensity of glioblastoma cells for B7-H3 (figure 5).

\section{DISCUSSION}

To the best of our knowledge, this is the first reported clinical trial that demonstrates that peroperative injection of the CTLA-4 blocking mAb ipilimumab plus the PD-1 blocking mAb nivolumab, following the resection of recurrent glioblastoma, is feasible, safe, and results in encouraging long-term OS.

The combination of an anti-PD-1 plus anti-CTLA-4 mAb (nivolumab plus ipilimumab) is approved for the treatment of melanoma, non-small cell lung cancer, hepatocellular carcinoma, mismatch repair deficient colorectal cancer, pleural mesothelioma, and renal cell carcinoma. Across tumor types, combination of both $\mathrm{mAb}$ resulted in a higher tumor response rate and improved survival when compared with monotherapy, at the cost of a higher incidence of irAE. ${ }^{43-49}$ Of note, the combination of ipilimumab and nivolumab was also found to have activity in patients with asymptomatic melanoma brain metastases. ${ }^{26}$
In patients with glioblastoma the combination of ipilimumab and nivolumab resulted in an unacceptable high incidence of irAE, with low antitumoral activity. ${ }^{18}$ Our study results indicate that the combination of nivolumab and ipilimumab can be locally administered in the brain tissue lining the glioblastoma resection cavity and this with a lower incidence of irAE as compared with intravenous administration. At the exception of a suspected higher incidence of transient low-grade postoperative fever, treatment was well tolerated. Low-grade, manageable irAE were seen in a minority of patients and study treatment was not stopped in any patient because of toxicity.

In the majority of patients with confirmed radiological progression during study treatment, subsequent progression eventually resulted in death despite salvage treatment with steroids, chemotherapy and VEGF(R) blocking agents. However, in one patient who developed progressive gadolinium-enhancing abnormalities early following study treatment, abnormalities slowly regressed, and this patient has become a long-term survivor, remaining progression-free 3 years after initiating study treatment. Additionally, tissue analysis in two patients who underwent resection of suspected tumor recurrences revealed no glioblastoma but post-treatment inflammatory changes. 

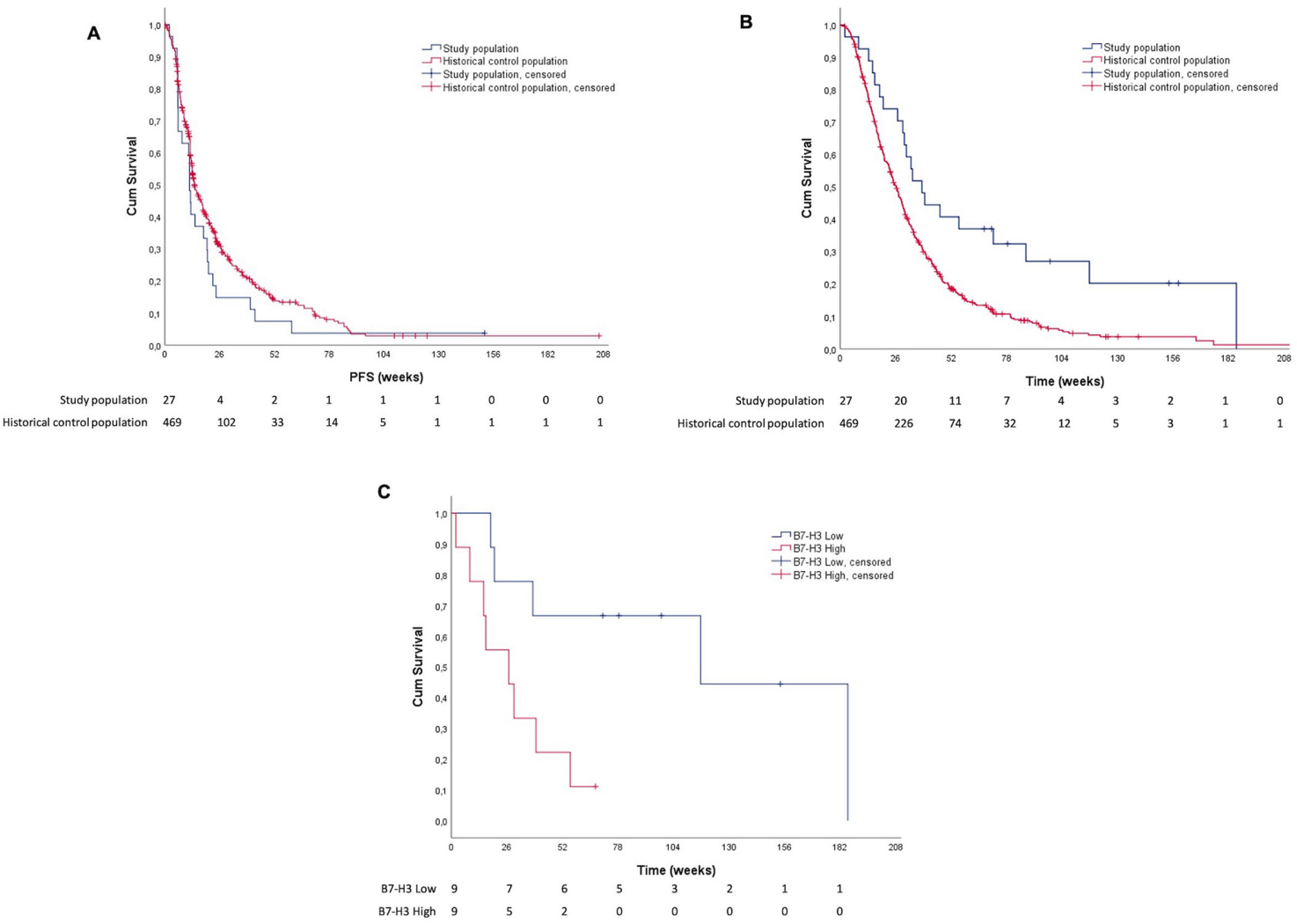

Figure 4 (A) Probability of progression-free survival according to Kaplan-Meier estimates for the study population $(n=27)$ and a pooled historical control population $(n=469)$ of Belgian patients with recurrent glioblastoma who were treated in three prospective phase II clinical trials and a multicenter medical need program for bevacizumab. (B) Probability of overall survival according to Kaplan-Meier estimates. (C) Probability for overall survival according to B7-H3 expression score by NanoString IO 360 gene expression profiling.

While insufficient tumor material from these patients was obtained to allow for in-depth analysis (eg, single-cell RNA-sequencing, and multiplexed immunofluorescence

\begin{tabular}{llr}
\hline Table 2 NGS data of resected tumor tissue & \\
\hline & & $\begin{array}{c}\text { N (\%) } \\
(\mathbf{n}=27)\end{array}$ \\
\hline Pathogenic mutations detected & Yes & $20(74)$ \\
& No & $7(26)$ \\
Pathogenic mutations & TERT c.-124C>T & $12(44)$ \\
& TP53 & $9(33)$ \\
& NF1 & $6(22)$ \\
& PTEN & $7(26)$ \\
& EGFR & $5(19)$ \\
& TERT c.-146C>T & $4(15)$ \\
& PIK3CA & $3(11)$ \\
ATRX & $3(11)$ \\
IDH1 R132H & $2(7)$ \\
IDH2 & $0(0)$
\end{tabular}

ATRX, gene encoding for transcriptional regulator ATRX; EGFR, epidermal growth factor receptor; IDH1, isocitrate dehydrogenase 1; IDH2, isocitrate dehydrogenase 2; NF1, neurofibromin 1; NGS, next-generation sequencing ; PIK3CA, phosphatidylinositol 3-kinase (PI3K) gene; PTEN, gene encoding for phosphatase and tensin homolog; TERT, telomerase reverse transcriptase; TP53, gene encoding for tumor protein p53. imaging) of potential secondary resistance mechanisms, this will be a valuable objective for the future.

In this clinical trial, an intravenous dose of nivolumab of $10 \mathrm{mg}$ was used to complement the intracranial injection. This dose is substantially lower than standard dosing. In melanoma patients who were treated with the same low dose of nivolumab with or without a single dose of ipilimumab in a prospective adjuvant phase II trial, TRAE were observed in $21(61 \%)$ and $17(77 \%)$ with $3(8 \%)$ and $1(4 \%)$ grade 3 irAE in patients treated with the lowdose ipilimumab plus nivolumab or low-dose nivolumab, respectively, indicating that this is a biologically active regimen.$^{50}$ Moreover, in a dose escalation phase I trial with nivolumab in patients with advanced melanoma, both ORR and irAE with a dose of nivolumab as low as $0.1 \mathrm{mg} /$ $\mathrm{kg}$ were in the same range as higher doses. ${ }^{51}$ Initial pharmacokinetic investigations also demonstrated durable PD-1 receptor occupancy in circulating $\mathrm{T}$ cells with a wide range of nivolumab doses. ${ }^{52}$ In this trial, we witnessed significantly lower numbers of AE compared with literature reports in patients with recurrent glioblastoma treated at full dose nivolumab. In the Checkmate-143 phase III trial, the incidence of nivolumab-related $\mathrm{AE}$ was $57 \%$ (any grade) with $18 \%$ grade $3 / 4 \mathrm{AE} .^{19}$

As we did not observe any dose-limiting toxicities, we considered the possibility of further increasing doses and 

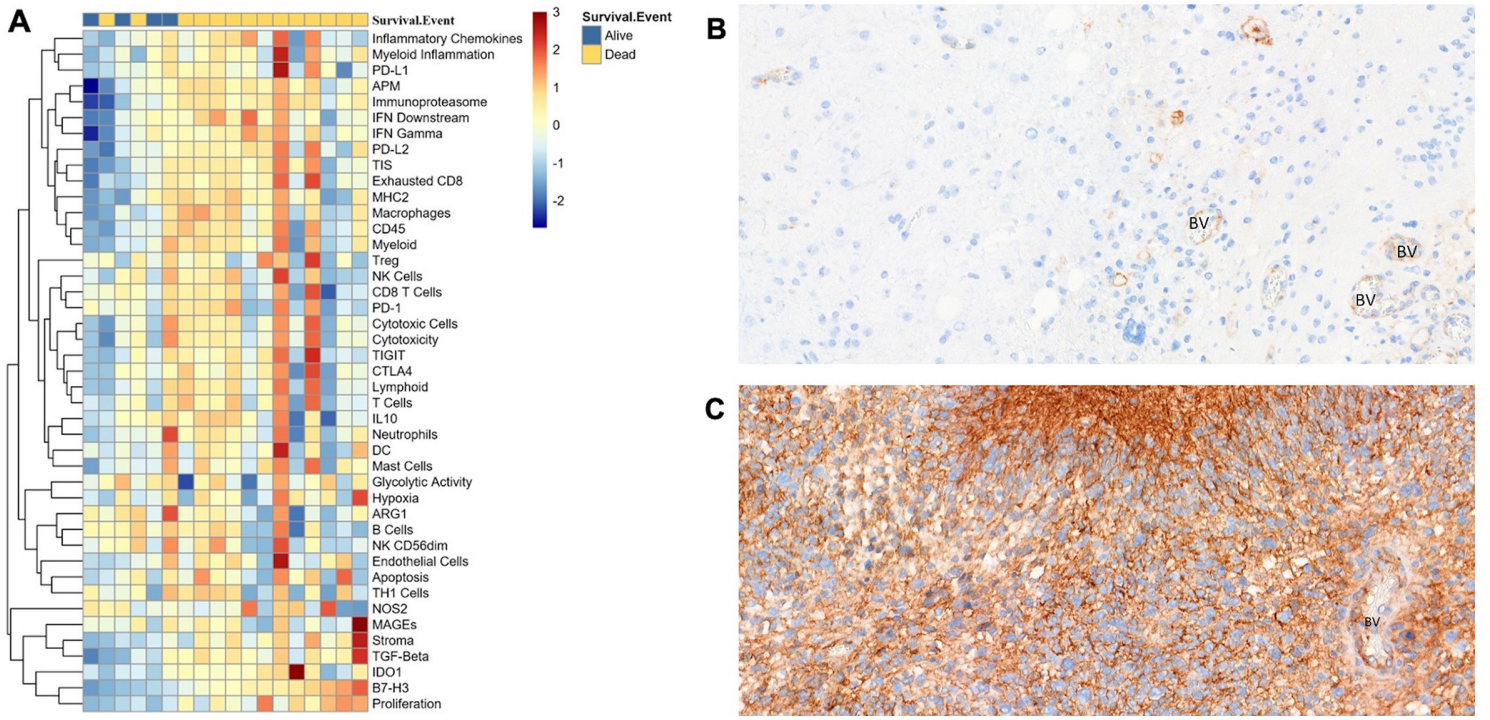

Figure 5 (A) Heat map representing NanoString 10360 gene expression profiling sorted by B7-H3 score (each vertical line represents the scores for one glioblastoma sample/patient). (B) Immunohistochemical (IHC) staining for B7-H3 of a gliobastoma with no staining of the tumor cells, the endothelial cells of the blood vessels (BV) are positive. (C) IHC staining for B7-H3 of a glioblastoma with strong staining of the tumor cells, the endothelial cells of the BV are also positive.

frequency of study drug administration. It was recently demonstrated that administration of checkpoint inhibition intrathecally through an Ommaya reservoir is safe in melanoma patients with leptomeningeal metastases. ${ }^{53}$ Currently, a combination of peroperative injection followed by intracavitary administration is under evaluation at our medical center. ${ }^{54}$ Experience with intratumoral administration of checkpoint inhibitors has also been made in patients with other solid tumors. In an exploratory phase I trial, autologous CD1c (BDCA1)+ myeloid dendritic cells isolated from the peripheral blood plus ipilimumab and avelumab (an anti-PD-L1 $\mathrm{mAb}$ ) were intratumorally injected. ${ }^{35} 55$ This approach was feasible and safe with mainly low-grade and manageable irAE. Antitumor responses were observed in injected as well as non-injected lesions. ${ }^{55}$ The addition of myeloid dendritic cells to intratumorally administered checkpoint inhibitors could also further enhance antitumoral activity in patients with recurrent glioblastoma.

Currently, there are no treatment options that have shown a survival benefit in patients diagnosed with recurrent glioblastoma emphasizing the need for new active treatment strategies. We found no indication of improved PFS in our phase I study patients compared with a large historical control group of patients who were treated with bevacizumab or the VEGFR-blocking tyrosine kinase inhibitor axitinib. Nevertheless, a favorable outcome in terms of OS of our phase I population compared with this same historical control group was found. ${ }^{494}$ It should be taken into consideration that the historical control population was uniformly treated with $\operatorname{VEGF}(\mathrm{R})$-blocking therapies. Such therapies have demonstrated to significantly improve PFS, while failing to improve OS. This may have influenced the comparison for the PFS endpoint. Differences in baseline prognostic characteristics should definitely be taken into account and restrain us from overinterpreting the OS comparison. Another limitation of this trial was that our study population was selected based on the resectability of the recurrent glioblastoma, and that patients did not have an extensive need for corticosteroid therapy. Previous reports, however, indicated that resection of recurrent disease prior to enrollment in phase II clinical trials may not influence OS. ${ }^{9}$ When compared with a subgroup of patients from the historical control population that was neither treated with corticosteroids at baseline, a significant OS benefit was also found. Of interest is the observation that the advantage in OS was most marked among patients with the longest survival suggesting a durable, rather than transient, survival advantage from the investigational immunotherapy. In an exploratory analysis, expression level of the suppressive immune checkpoint receptor B7-H3 was found to strongly and independently correlate with OS. As B7-H3 has previously been reported to play a role in glioblastoma immunobiology, this receptor deserves further study as a potential biomarker for glioblastoma immunotherapy. ${ }^{5657}$

Our investigated doses of nivolumab and ipilimumab are substantially lower compared with standard dosing at $1 \mathrm{mg} / \mathrm{kg}$ and $3 \mathrm{mg} / \mathrm{kg}$, respectively. Thereby, the cost could be considerably decreased. The unique approach of peroperative injection of immune checkpoint blocking mAb locally in the brain tissue offers the possibility for a low-cost immunotherapeutic backbone with acceptable toxicity that could be combined with additional immunotherapeutic interventions to further improve the outcome of patients with recurrent glioblastoma. 


\section{Author affiliations}

${ }^{1}$ Department of Neurosurgery, Vrije Universiteit Brussel (VUB), Universitair

Ziekenhuis Brussel (UZ Brussel), Brussels, Belgium

${ }^{2}$ Department of Medical Oncology, Vrije Universiteit Brussel (VUB), Universitair

Ziekenhuis Brussel (UZ Brussel), Brussels, Belgium

${ }^{3}$ Centre for Medical Genetics, Vrije Universiteit Brussel (VUB), Universitair Ziekenhuis Brussel (UZ Brussel), Brussels, Belgium

${ }^{4}$ Department of Neurology, Vrije Universiteit Brussel (VUB), Universitair Ziekenhuis Brussel (UZ Brussel), Brussels, Belgium

${ }^{5}$ Department of Pathology, Vrije Universiteit Brussel (VUB), Universitair Ziekenhuis Brussel (UZ Brussel), Brussels, Belgium

${ }^{6}$ Department of Pathology, Erasme Hospital, Université Libre de Bruxelles, Brussels, Belgium

${ }^{7}$ Cell Carta NV, Antwerp, Belgium

${ }^{8}$ Department of Radiology, Vrije Universiteit Brussel (VUB), Universitair Ziekenhuis Brussel (UZ Brussel), Brussels, Belgium

${ }^{9}$ Department of Nuclear Medicine, Vrije Universiteit Brussel (VUB), Universitair Ziekenhuis Brussel (UZ Brussel), Brussels, Belgium

Acknowledgements We would like to acknowledge the patients who consented to participate in this study, their families, and professional healthcare providers. We would also like to thank the study nurses Katrien Van den Bossche and Maud Allard (UZ Brussel) for their help with the data collection.

Contributors BN designed the study and drafted the clinical protocol. BN, JKS, and JD did the acquisition, analysis, and interpretation of the data. JD performed the neurosurgical intervention. JD, JKS, GA, SK, LS, and BN provided care for study patients. CB helped with data acquisition. A-MVB and HE performed analysis of medical imaging. AM performed the neuropathological analysis. RF and LC assessed the PD-L1 and CD8 expression, MK did the B7-H3 IHC analysis. BC, SVD, and FV performed next-generation sequencing and preparation of samples for NanoString analysis. All authors reviewed the manuscript. Final manuscript was read and approved for submission by all authors.

Funding This study was sponsored and funded by the Universitair Ziekenhuis Brussel. The corresponding author had full access to all the data in the study and had final responsibility for the decision to submit for publication.

Competing interests Julia Katharina Schwarze reports non-financial support from MSD and Amgen; grants for research from Kom op tegen Kanker, Stichting tegen Kanker. Gil Awada reports grants and nonfinancial support from Pfizer, Novartis; non-financial support from MSD, Astellas; personal fees from Novartis; grants from Kom op tegen Kanker, Stichting tegen Kanker. Bart Neyns reports personal financial compensation from Roche, Bristol-Myers Squibb, Merck Sharp \& Dohme, Novartis, AstraZeneca for public speaking, consultancy and participation in advisory board meeting. The institution (UZ Brussel) received research funding related to research projects conducted by Bart Neyns from Pfizer, Novartis, Roche, Merck-Serono. The other authors do not declare any competing interests.

\section{Patient consent for publication Not required.}

Provenance and peer review Not commissioned; externally peer reviewed.

Data availability statement All data relevant to the study are included in the article or uploaded as supplementary information.

Supplemental material This content has been supplied by the author(s). It has not been vetted by BMJ Publishing Group Limited (BMJ) and may not have been peer-reviewed. Any opinions or recommendations discussed are solely those of the author(s) and are not endorsed by BMJ. BMJ disclaims all liability and responsibility arising from any reliance placed on the content. Where the content includes any translated material, BMJ does not warrant the accuracy and reliability of the translations (including but not limited to local regulations, clinical guidelines, terminology, drug names and drug dosages), and is not responsible for any error and/or omissions arising from translation and adaptation or otherwise.

Open access This is an open access article distributed in accordance with the Creative Commons Attribution Non Commercial (CC BY-NC 4.0) license, which permits others to distribute, remix, adapt, build upon this work non-commercially, and license their derivative works on different terms, provided the original work is properly cited, appropriate credit is given, any changes made indicated, and the use is non-commercial. See http://creativecommons.org/licenses/by-nc/4.0/.

\section{ORCID iDs}

Julia Katharina Schwarze http://orcid.org/0000-0001-8990-5380

Gil Awada http://orcid.org/0000-0001-9535-0430
Wietse Geens http://orcid.org/0000-0002-2496-9209

Bart Neyns http://orcid.org/0000-0003-0658-5903

\section{REFERENCES}

1 Stupp R, Hegi ME, Mason WP, et al. Effects of radiotherapy with concomitant and adjuvant temozolomide versus radiotherapy alone on survival in glioblastoma in a randomised phase III study: 5-year analysis of the EORTC-NCIC trial. Lancet Oncol 2009;10:459-66.

2 Wick W, Gorlia T, Bendszus M, et al. Lomustine and bevacizumab in progressive glioblastoma. N Engl J Med 2017;377:1954-63.

3 Batchelor TT, Mulholland P, Neyns B, et al. Phase III randomized trial comparing the efficacy of cediranib as monotherapy, and in combination with lomustine, versus lomustine alone in patients with recurrent glioblastoma. J Clin Oncol 2013;31:3212-8.

4 Wong ET, Hess KR, Gleason MJ, et al. Outcomes and prognostic factors in recurrent glioma patients enrolled onto phase II clinical trials. J Clin Oncol 1999;17:2572-8.

5 Lamborn KR, Yung WKA, Chang SM, et al. Progression-Free survival: an important end point in evaluating therapy for recurrent high-grade gliomas. Neuro Oncol 2008;10:162-70.

6 Oppenlander ME, Wolf AB, Snyder LA, et al. An extent of resection threshold for recurrent glioblastoma and its risk for neurological morbidity. J Neurosurg 2014;120:846-53.

7 Ringel F, Pape H, Sabel M, et al. Clinical benefit from resection of recurrent glioblastomas: results of a multicenter study including 503 patients with recurrent glioblastomas undergoing surgical resection. Neuro Oncol 2016;18:96-104.

8 Bloch O, Han SJ, Cha S, et al. Impact of extent of resection for recurrent glioblastoma on overall survival: clinical article. $J$ Neurosurg 2012;117:1032-8.

9 Clarke JL, Ennis MM, Yung WKA, et al. Is surgery at progression a prognostic marker for improved 6-month progression-free survival or overall survival for patients with recurrent glioblastoma? Neuro Oncol 2011;13:1118-24.

10 Ribas A, Wolchok JD. Cancer immunotherapy using checkpoint blockade. Science 2018;359:1350-5.

11 Agarwalla P, Barnard Z, Fecci $P$, et al. Sequential immunotherapy by vaccination with GM-CSF-expressing glioma cells and CTLA-4 blockade effectively treats established murine intracranial tumors. $J$ Immunother 2012;35:385-9.

12 Fecci PE, Ochiai H, Mitchell DA, et al. Systemic CTLA-4 blockade ameliorates glioma-induced changes to the CD4+ T cell compartment without affecting regulatory T-cell function. Clin Cancer Res 2007;13:2158-67.

13 Antonios JP, Soto H, Everson RG, et al. Pd-1 blockade enhances the vaccination-induced immune response in glioma. JCl Insight 2016;1.

14 Reardon DA, Gokhale PC, Klein SR, et al. Glioblastoma eradication following immune checkpoint blockade in an orthotopic, immunocompetent model. Cancer Immunol Res 2016;4:124-35.

15 Wainwright DA, Chang AL, Dey M, et al. Durable therapeutic efficacy utilizing combinatorial blockade against IDO, CTLA-4, and PD-L1 in mice with brain tumors. Clin Cancer Res 2014;20:5290-301.

16 Zeng J, See AP, Phallen J, et al. Anti-PD-1 blockade and stereotactic radiation produce long-term survival in mice with intracranial gliomas. Int J Radiat Oncol Biol Phys 2013;86:343-9.

17 Kim JE, Patel MA, Mangraviti A, et al. Combination therapy with antiPD-1, Anti-TIM-3, and focal radiation results in regression of murine gliomas. Clin Cancer Res 2017;23:124-36.

18 Omuro A, Vlahovic G, Lim M, et al. Nivolumab with or without ipilimumab in patients with recurrent glioblastoma: results from exploratory phase I cohorts of CheckMate 143. Neuro Oncol 2018:20:674-86.

19 Reardon DA, Brandes AA, Omuro A, et al. Effect of nivolumab vs bevacizumab in patients with recurrent glioblastoma: the CheckMate 143 phase 3 randomized clinical trial. JAMA Oncol 2020;6:10031010.

20 Blumenthal DT, Yalon M, Vainer GW, et al. Pembrolizumab: first experience with recurrent primary central nervous system (CNS) tumors. J Neurooncol 2016;129:453-60.

21 Reardon DA, Nayak L, Peters KB, et al. Phase II study of pembrolizumab or pembrolizumab plus bevacizumab for recurrent glioblastoma (rGBM) patients. Journal of Clinical Oncology 2018;36:2006-06.

22 Sampson JH, Omuro AMP, Preusser M, et al. A randomized, phase 3 , open-label study of nivolumab versus temozolomide (TMZ) in combination with radiotherapy (RT) in adult patients (pts) with newly diagnosed, O-6-methylguanine DNA methyltransferase (MGMT)unmethylated glioblastoma (GBM): CheckMate-498. Journal of Clinical Oncology 2016;34:TPS2079-TPS79. 
23 Cristescu R, Mogg R, Ayers M, et al. Pan-tumor genomic biomarkers for PD-1 checkpoint blockade-based immunotherapy. Science 2018;362. doi:10.1126/science.aar3593. [Epub ahead of print: 1210 2018].

24 Sampson JH, Gunn MD, Fecci PE, et al. Brain immunology and immunotherapy in brain tumours. Nat Rev Cancer 2020;20:12-25.

25 Bouffet E, Larouche V, Campbell BB, et al. Immune checkpoin inhibition for Hypermutant glioblastoma multiforme resulting from germline biallelic mismatch repair deficiency. J Clin Oncol 2016;34:2206-11.

26 Tawbi HA, Forsyth PA, Algazi A, et al. Combined nivolumab and ipilimumab in melanoma metastatic to the brain. N Engl J Med 2018;379:722-30.

27 Chongsathidkiet P, Jackson C, Koyama S, et al. Sequestration of T cells in bone marrow in the setting of glioblastoma and other intracranial tumors. Nat Med 2018;24:1459-68.

28 Mohme M, Schliffke S, Maire CL, et al. Immunophenotyping of newly diagnosed and recurrent glioblastoma defines distinct immune exhaustion profiles in peripheral and tumor-infiltrating lymphocytes. Clin Cancer Res 2018;24:4187-200.

29 de Groot J, Penas-Prado M, Alfaro-Munoz K, et al. Window-ofopportunity clinical trial of pembrolizumab in patients with recurrent glioblastoma reveals predominance of immune-suppressive macrophages. Neuro Oncol 2020;22:539-49.

30 Cloughesy TF, Mochizuki AY, Orpilla JR, et al. Neoadjuvant anti-PD-1 immunotherapy promotes a survival benefit with intratumoral and systemic immune responses in recurrent glioblastoma. Nat Med 2019;25:477-86.

31 Schalper KA, Rodriguez-Ruiz ME, Diez-Valle R, et al. Neoadjuvant nivolumab modifies the tumor immune microenvironment in resectable glioblastoma. Nat Med 2019;25:470-6.

32 Marabelle A, Kohrt H, Levy R. Intratumoral anti-CTLA-4 therapy: enhancing efficacy while avoiding toxicity. Clin Cancer Res 2013;19:5261-3.

33 Fransen MF, van der Sluis TC, Ossendorp F, et al. Controlled local delivery of CTLA-4 blocking antibody induces CD8+ T-celldependent tumor eradication and decreases risk of toxic side effects. Clin Cancer Res 2013;19:5381-9.

34 Ray A, Williams MA, Meek SM, et al. A phase I study of intratumoral ipilimumab and interleukin-2 in patients with advanced melanoma. Oncotarget 2016;7:64390-64399.

35 Schwarze JK, Awada G, Van Riet I, Riet V I, et al. A phase I clinical trial on intratumoral administration of autologous CD1c (BDCA1 )+ myeloid dendritic cells (myDC) in combination with ipilimumab (IPI) and avelumab (AVE) plus intravenous low-dose nivolumab (NIVO) in patients with advanced solid tumors. Annals of Oncology 2018:29:x14.

36 Ogino S, Kawasaki T, Brahmandam M, et al. Precision and performance characteristics of bisulfite conversion and real-time PCR (MethyLight) for quantitative DNA methylation analysis. J Mol Diagn 2006;8:209-17.

37 Eads CA, Danenberg KD, Kawakami K, et al. MethyLight: a highthroughput assay to measure DNA methylation. Nucleic Acids Res 2000;28:E32.

38 Froyen G, Le Mercier M, Lierman E, et al. Standardization of somatic variant classifications in solid and haematological tumours by a twolevel approach of biological and clinical classes: an initiative of the Belgian ComPerMed expert panel. Cancers 2019;11. doi:10.3390/ cancers11122030. [Epub ahead of print: 1612 2019].

39 Duerinck J, Clement PM, Bouttens F, et al. Patient outcome in the Belgian medical need program on bevacizumab for recurrent glioblastoma. J Neurol 2015;262:742-51.

40 Duerinck J, Du Four S, Bouttens F, et al. Randomized phase II trial comparing axitinib with the combination of axitinib and lomustine in patients with recurrent glioblastoma. J Neurooncol 2018;136:115-25.

41 Duerinck J, Du Four S, Vandervorst F, et al. Randomized phase II study of axitinib versus physicians best alternative choice of therapy in patients with recurrent glioblastoma. $J$ Neurooncol 2016:128:147-55.

42 Awada G, Ben Salama L, De Cremer J, et al. Axitinib plus avelumab in the treatment of recurrent glioblastoma: a stratified, open-label, single-center phase 2 clinical trial (GliAvAx). J Immunother Cancer 2020;8:e001146

43 Hellmann MD, Ciuleanu T-E, Pluzanski A, et al. Nivolumab plus ipilimumab in lung cancer with a high tumor mutational burden. $N$ Engl J Med 2018;378:2093-104.

44 Hellmann MD, Paz-Ares L, Bernabe Caro R, et al. Nivolumab plus ipilimumab in advanced non-small-cell lung cancer. N Engl J Med 2019;381:2020-31.

45 Larkin J, Chiarion-Sileni V, Gonzalez R, et al. Five-Year survival with combined nivolumab and ipilimumab in advanced melanoma. $N$ Engl J Med 2019;381:1535-46.

46 Motzer RJ, Tannir NM, McDermott DF, et al. Nivolumab plus ipilimumab versus sunitinib in advanced renal-cell carcinoma. $N$ Engl J Med 2018;378:1277-90.

47 Scherpereel A, Mazieres J, Greillier L, et al. Nivolumab or nivolumab plus ipilimumab in patients with relapsed malignant pleural mesothelioma (IFCT-1501 MAPS2): a multicentre, openlabel, randomised, non-comparative, phase 2 trial. Lancet Oncol 2019;20:239-53.

48 Yau T, Kang Y-K, Kim T-Y, et al. Efficacy and safety of nivolumab plus ipilimumab in patients with advanced hepatocellular carcinoma previously treated with sorafenib: the CheckMate 040 randomized clinical trial. JAMA Oncol 2020;6:e204564.

49 Overman MJ, Lonardi S, Wong KYM, et al. Durable clinical benefit with nivolumab plus ipilimumab in DNA mismatch Repair-Deficient/ Microsatellite Instability-High metastatic colorectal cancer. J Clin Oncol 2018;36:773-9.

50 Julia Katharina Schwarze VVGAYJTSBN, Universitair Ziekenhuis Brussel BB, eds. A sequential dual cohort phase I/ clinical trial on adjuvant low-dose nivolumab with or without low-dose ipilimumab as adjuvant therapy following the resection of melanoma macrometastases $(\mathrm{Mm})$ ASCO annual meeting. Alexandria, Virginia: American Society of Clinical Oncology, 2019.

51 Topalian SL, Sznol M, McDermott DF, et al. Survival, durable tumor remission, and long-term safety in patients with advanced melanoma receiving nivolumab. J Clin Oncol 2014;32:1020-30.

52 Brahmer JR, Drake CG, Wollner I, et al. Phase I study of single-agent anti-programmed death-1 (MDX-1106) in refractory solid tumors: safety, clinical activity, pharmacodynamics, and immunologic correlates. J Clin Oncol 2010;28:3167-75.

53 Glitza IC, Rohlfs ML, lqbal M, et al. A phase I/lb study of concurrent intravenous (IV) and intrathecal (it) nivolumab (Nivo) for melanoma patients (PTS) with leptomeningeal disease (LMD). Annals of Oncology 2018;29:viii464.

54 Julia Katharina Schwarze JDIDGASKLFLSFVARHEA-MVAMBN, Department of Medical Oncology UZBBB, et al, eds. A phase I clinical trial on intratumoral and intracavitary administration of ipilimumab and nivolumab in patients with recurrent glioblastoma; ASCO virtual scientific program. Alexandria, Virginia: American Society of Clinical Oncology, 2020.

55 Schwarze JK, Awada G, Cras L, et al. Intratumoral Combinatorial Administration of CD1c (BDCA-1) $)^{+}$Myeloid Dendritic Cells Plus Ipilimumab and Avelumab in Combination with Intravenous LowDose Nivolumab in Patients with Advanced Solid Tumors: A Phase IB Clinical Trial. Vaccines 2020;8. doi:10.3390/vaccines8040670. [Epub ahead of print: 10112020 ]

56 Zhang C, Zhang Z, Li F, et al. Large-Scale analysis reveals the specific clinical and immune features of $\mathrm{B} 7-\mathrm{H} 3$ in glioma. Oncoimmunology 2018;7:e1461304.

57 Yang S, Wei W, Zhao Q. B7-H3, a checkpoint molecule, as a target for cancer immunotherapy. Int J Biol Sci 2020;16:1767-73. 